\title{
Risk of infection in primary, elective total hip arthroplasty with direct anterior approach or lateral transgluteal approach: a prospective cohort study of 1104 hips
}

\author{
Thomas Ilchmann ${ }^{1,2}$, Werner Zimmerli ${ }^{1}$, Lilianna Bolliger ${ }^{3}$, Peter Graber ${ }^{1}$ and Martin Clauss ${ }^{1,3^{*}}$
}

\begin{abstract}
Background: The direct anterior approach (DAA) is increasingly popular for hip replacement. However, the small incision and the location near to the groin might increase the risk of periprosthetic joint infection (PJI). We asked the questions (i) whether there is an increased risk of infection for this approach, and (ii) whether the spectrum of microorganisms differs between patients with DAA and those with lateral transgluteal approach (LAT).

Methods: All patients operated between 08/2006 and 12/2013 were followed prospectively in an in house register. The DAA was introduced as routine in 02/2009 at our hospital. Patients with primary elective hip replacement without previous operations were included. Follow-up was scheduled after 6,12 weeks and 1, 2 years. PJI was defined according to standardized criteria.

Results: One thousand one hundred four patients were studied, 700 were operated with DAA and 404 with LAT. No patient was lost to follow-up. PJI was diagnosed in 23/1104 (2.1\%) patients, 16 (2.3 \%) in the group with DAA, and $7(1.7 \%)$ in the group with LAT. Patients with infection had a higher BMI $(p<0.001)$ and a higher ASA score $(p<0.001)$. Only patients with the DAA had exogenous PJI caused by gramnegative bacilli ( $35.7 \%$ vs $0 \%, p=0.26$ ). In the DAA-group, the fraction of patients with polymicrobial infection was somewhat higher than in the LAT-group ( $50 \%$ vs $33 \%, P=0.64$ ).
\end{abstract}

Conclusion: There was no increased risk of infection for the DAA.

Keywords: PJI, Direct anterior approach, Lateral transgluteal approach, Total hip arthroplasty, Prosthetic joint infection

\section{Background}

Periprosthetic joint infection (PJI) is one of the major complications after total hip arthroplasty (THA), with a reported incidence of 0.5 to $3 \%$ [1]. Many factors contribute to the risk of PJI, some of them can be influenced by appropriate measures [2]. Recently, an increasing risk of PJI has been described in the Nordic hip registers [3] and from the US [4]. The reported infection rates depend on the definition of infection, the awareness of the surgeon, the quality of reporting, and the

\footnotetext{
* Correspondence: martin.clauss@ksbl.ch

${ }^{1}$ Interdisciplinary Unit for Orthopedic Infections, Kantonsspital Baselland, Rheinstrasse 26, 4410 Liestal, Switzerland

${ }^{3}$ Department for Orthopedics and Trauma Surgery, Kantonsspital Baselland, Rheinstrasse 26, 4410 Liestal, Switzerland

Full list of author information is available at the end of the article
}

length of follow-up. In implant registers, there might be confounding factors, and data might be less reliable as compared to specific prospective infection registration $[3,5,6]$.

The surgical approach affects the posture of the patient, the effectiveness of laminar air flow and the draping [7]. In addition, skin quality including the type of the microbiome differs at the various incision sites [8]. Thus, the surgical approach itself might influence the rate of PJI, as well as the spectrum of infecting microorganisms. For the posterior and lateral approaches, such a difference could not be shown [9]. However, there is an increasing interest in the direct anterior approach (DAA), being assumed to be more anatomical and thus less 
invasive. For the DAA, the incision is closer to the groin (Fig. 1), which is a highly colonized region.

It has been shown, that groin incision is a risk factor for vascular graft associated infections [10]. Furthermore, aiming to shorter incisions might be a potential risk of increased skin irritation by traction and by surgical instruments. This might cause problems with wound healing or contamination of the implant with consecutive PJI [11-13].

In our hospital, we introduced DAA according to Matta [14] as routine in 2009 for primary elective total hip replacement [15]. Three years after introduction we observed a clustering of early infections caused either by gram-negative bacilli or by multiple microorganisms (polymicrobial). Thus, we asked the question as to whether there is (i) an increased risk of infection for DAA, and (ii) whether the spectrum of microorganisms differs between patients with DAA and those with the lateral transgluteal approach (LAT), being routine at our hospital until 2009.

\section{Methods}

This is a retrospective observational study with prospectively acquired data of a cohort from our University affiliated academic teaching hospital with an interdisciplinary unit for orthopedic infections. This study was approved by the local Ethics Committee (Northwest and Central Switzerland, EKNZ 2015-426). In accordance with the Ethics Committee, no specific informed consent was required.

Between 08/2006 and 12/2013, 1280 primary THA were performed. Only elective hip replacements without previous hip surgery were included in the study. In total, 176 hips were excluded, 86 had a hip fracture, 78 had previous hip surgery, 11 had bone metastases, and one had rapid destructive inflammation. Out of the 1104

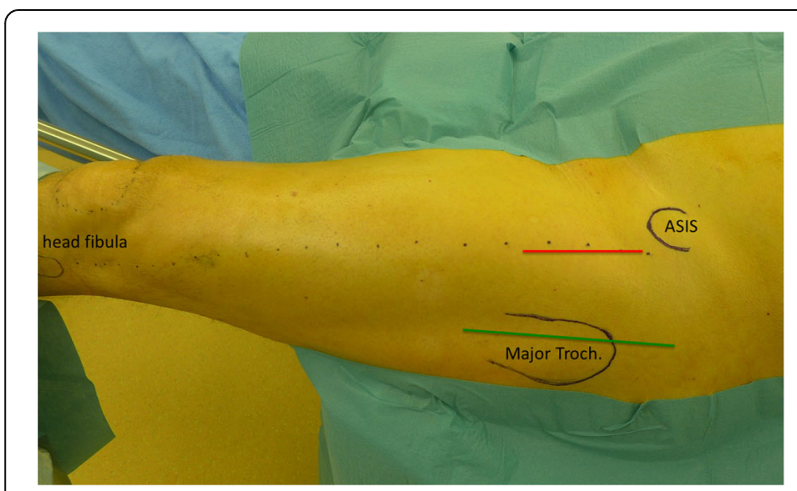

Fig. 1 For the DAA (red line) a straight incision following the proximal part of a line (dotted) between the anterior superior iliac spine (ASIS) and the head of the fibula was made. For the LAT (green line) a straight incision was used parallel to the femur in the middle of the major trochanter included hips, 700 (63\%) were operated with DAA, and 404 with LAT (Table 1).

The lateral approach in supine position (Fig. 1) was exclusively used until 3/2009, i.e. during the first 31 months of the study. Thereafter, the DAA was introduced. The first 100 DAA interventions were performed by 2 experienced hip surgeons. Later young consultants and residents were trained as it was standard for LAT too. After introduction of DAA, 34 hips were still operated via LAT for various reasons. Eight had previous surgery on the opposite via a lateral approach and requested the same approach. In 7 hips, DAA was not performed because of skin irritations in the groin, in 6 because of obesity, in 4 because of severe flexion contracture or a repair of the gluteal muscles, in 5 for teaching reasons, in one because of hip dysplasia and a deficient acetabular roof, and in three because no DAA trained surgeon was available.

For the DAA, the patients were postured in supine position on a trauma table [14]. We used the direct anterior approach (Fig. 1) according to Smith-Peterson [16] with a split of the fascia of the tensor fascia lata and preparation through the intramuscular plane between $M$. sartorius and $M$. tensor fascia latae [15]. Patients operated from lateral [17] were posed in supine position, the peri- and postoperative setting was the same.

All interventions were performed in laminar airflow without protective helmets. Patients were covered with forced air flow blankets. Single shot antibiotic prophylaxis (cefuroxime $1.5 \mathrm{~g}$ ) was administered 30-60 minutes prior to skin incision. The skin was disinfected three times with a povidone-iodine solution (Betaseptic ${ }^{\mathrm{TM}}$, Mundipharma, Basel, $\mathrm{CH}$ ) for totally 5 minutes [18]. Afterwards, in the DAA group, a plastic trauma draping (3 M, Rüschlikon, $\mathrm{CH}$ ) was used, around the planned skin incision, the drape was removed and the skin was disinfected once more. The LAT group was operated without a plastic draping. Two pairs of gloves were used, before start of surgery the outer pair of gloves was changed. Perioperatively, the wound was cleaned and disinfected with a $0.1 \%$ polyhexanidesolution (Lavasept ${ }^{\mathrm{TM}}$ BBraun, Sempach, CH). The skin was closed with subcutaneous and cutaneous sutures.

Sutures were removed by the family practitioner or by the rehabilitation staff 12-14 days after surgery. Outpatient physicians were informed to resubmit the patient without prescribing antibiotics in case of any wound healing disturbance (redness, dehiscence, oozing).

The most frequently used implants were spherical pressfit cups and cemented or uncemented stems (Table 2).

Body mass index (BMI), American Society of Anesthesiologist (ASA) score, procedure time and blood-loss were taken from the charts. All patients were prospectively followed in our in-house register, which has been introduced in 1984. There was an approval of the local ethical authorities for follow-up visits (EKNZ 
Table 1 Study population

\begin{tabular}{lllll}
\hline & DAA & LAT & Total & $p$ \\
& $n=700(63 \%)$ & $n=404(37 \%)$ & $n=1104$ & \\
\hline Age (median, SD) & $71(10.6)$ & $71( \pm 10.6)$ & $71( \pm 10.6)$ & n.s. \\
Male & $369(53 \%)$ & $212(53 \%)$ & $581(53 \%)$ & n.s. \\
BMI (mean, SD) & $26.6(4.2)$ & $27.2(5.2)$ & $26.8(4.6)$ & 0.009 \\
ASA I and II & $575(82 \%)$ & $338(84 \%)$ & $913(83 \%)$ & n.s. \\
Primary & $644(92 \%)$ & $375(93 \%)$ & $1019(92 \%)$ & n.s. \\
Osteoarthritis & & & & \\
Head necrosis & $44(6 \%)$ & $16(4 \%)$ & $60(5 \%)$ & \\
Inflammation (RA) & $7(1 \%)$ & $2(0.5 \%)$ & $9(0.8 \%)$ & \\
Other indications & $5(1 \%)$ & $11(3 \%)$ & $16(2 \%)$ & \\
\hline DAA dirt an $\%)$
\end{tabular}

$D A A$ direct anterior approach, $L A T$ lateral approach, $B M I$ body mass index $(\mathrm{kg} / \mathrm{m} 2)$, ASA American Society of Anesthesiologist, RA rheumatoid arthritis

2015-426). Follow-up was scheduled at 6 and 12 weeks, and after 1, 2 and 5 years. A standardized clinical and radiological follow-up protocol was followed, including registration of any adverse events [15]. All patients were seen at every follow-up by one out of 3 consultants of the hip unit.

PJI was diagnosed according to the IDSA-guidelines [19]. At least one of the following criteria had to be fulfilled: (a) presence of a sinus tract, (b) visible pus surrounding the joint without other explanation (e.g. no crystals), (c) acute inflammation on histopathological examination ( $>5$ neutrophils/high-power field), (d) $>4200$ leukocyte per $\mu \mathrm{l}$ and/or $>80 \%$ polymorphonuclear leukocytes in synovial fluid, (e) growth of the same microorganism in at least two cultures of synovial fluid, peri-prosthetic tissue and/or sonication fluid. Patients were classified into acute postoperative ( $\leq 1$ month after implantation), acute hematogenous ( $\leq 3$ weeks of infectious symptoms), and chronic PJI (all other situations) [20]. As a rule, patients with PJI were managed according to an established algorithm

Table 2 Type of implants

\begin{tabular}{llll}
\hline & Implant & Number & Percent \\
\hline Cups & RM and RM vitamys (Mathys) & 615 & $56 \%$ \\
& Selexys TH+ (Mathys) & 174 & $16 \%$ \\
& Allofit (Zimmer) & 261 & $24 \%$ \\
& PE cem & 24 & $2 \%$ \\
& Others & 30 & $3 \%$ \\
Uncemented stems & TwinSys (Mathys) & 400 & $36 \%$ \\
& Avenir (Zimmer) & 84 & $8 \%$ \\
& CBC (Mathys)/CLS (Zimmer) & 182 & $16 \%$ \\
Cemented stems & TwinSys (Mathys) & 272 & $36 \%$ \\
& CCA (Mathys)/Muller & 166 & $15 \%$ \\
& Straight Stem & & \\
\hline
\end{tabular}

[21] with debridement and implant retention (DAIR), one-stage or two-stage procedures, respectively [22].

\section{Statistics}

A Shapiro-Wilk test for normality was performed to determine whether continuous data were normally distributed. All data were non-normal distributed and were presented as median and range. Mann Whitney rank sum test was used for comparison of two continuous (but non-normal distributed) variables, Chi Square or exact Fisher test for comparison of categorical variables. Conclusions about ASA Score were considered with regard to two consolidated classes: First class includes ASA Score I and II versus second class with ASA Score III and IV. A $P$-value of $\leq 0.05$ was considered to be statistically significant. Data were analyzed using IBM SPSS Statistics Version 23.

\section{Results}

The minimum follow-up was two years; no patient was lost to follow-up. None of the patients was treated with antibiotics for suspected PJI before evaluation by the interdisciplinary infection team. Of all 1104 THA, 23 (2.1\%) had PJI during the follow-up period.

Acute postoperative PJI (median 17 days, range 13 to 30 days postoperative) was diagnosed in 11 THA, 8 in the DAA- and 3 in the LAT-group. Three THA had acute hematogenous PJI $(3.5,5$ and 8 months, respectively, after implantation). All THA with acute PJI were treated with DAIR, mostly with exchange of the modular parts of the device. Nine THA had chronic PJI (median 11.4 , range 1.5 to 47 months after implantation). Two were treated with DAIR within the first 50 days after implantation, five were treated with one-stage exchange and two with two-stage exchange. At the final follow-up, no patient with treatment for PJI had signs of persistence of infection (Table 3).

As we wanted to analyze, whether there is an increased risk for exogenous PJI with the DAA, we excluded the three cases with hematogenous PJI (2 DAA, 1 LAT) from further analysis. The rate of exogenous PJI was similar in both groups, namely 14/700 (2.0 \%) in the DAA- and 6/ $404(1.5 \%)$ in the LAT-group $(P=0.54)$. Five of the 34 patients treated with the LAT-approach during the DAAperiod suffered from a PJI; one was operated from lateral due to severe adipositas (BMI $45 \mathrm{~kg} / \mathrm{m} 2$, UPI 883), three due to skin irritations in the groin making an DAA approach impossible (UPI 599, 1100, 1121), and one because no DAA trained surgeon was available (UPI 453). Table 3 summarizes patient characteristics, microorganisms, type of infection and surgical treatment of all patients with PJI. The spectrum of microorganisms was different in the two groups. Only patients with the DAA had PJI caused by gramnegative bacilli $(5 / 14$ vs $0 / 6, P=0.26)$. 
Table 3 Patient characteristics, microorganisms, type of infection and surgical treatment of all episodes with PJI

\begin{tabular}{|c|c|c|c|c|c|c|c|c|c|}
\hline UPI & Age & Gender & $\mathrm{BMI}$ & ASA & Approach & Microorganisms & Type of PJI & $\begin{array}{l}\text { Time after } \\
\text { implantation [d] }\end{array}$ & Treatment \\
\hline 874 & 83 & male & 34.3 & 3 & DAA & S. epidermidis, Proteus mirabilis & 1 & 13 & DAIR \\
\hline 873 & 73 & female & 33.9 & 2 & DAA & $\begin{array}{l}\text { S. epidermidis, Morganella morganii } \\
\text { Klebsiella pneumoniae, Pseudomonas } \\
\text { aeruginosa, E. coli }\end{array}$ & 1 & 15 & DAIR \\
\hline 1028 & 56 & male & 32.4 & 2 & DAA & S. aureus & 1 & 15 & DAIR \\
\hline 987 & 77 & male & 33.8 & 3 & DAA & $\begin{array}{l}\text { S. epidermidis, S. haemolyticus, } \\
\text { Aerococcus sp., Acinebacter sp. }\end{array}$ & 1 & 18 & DAIR \\
\hline 812 & 66 & male & 29.0 & 3 & DAA & S. epidermidis & 1 & 20 & DAIR \\
\hline 709 & 68 & female & 37.7 & 2 & DAA & S. epidermidis & 1 & 21 & DAIR \\
\hline 932 & 88 & male & 26.0 & 3 & DAA & S. aureus, Citrobacter freundii & 1 & 28 & DAIR \\
\hline 978 & 71 & female & 36.8 & 3 & DAA & S. epidermidis & 1 & 30 & DAIR \\
\hline 840 & 76 & female & 37.8 & 3 & DAA & $\begin{array}{l}\text { S. epidermidis, Gr.B Streptococcus, } \\
\text { Morganella morganii }\end{array}$ & 3 & 50 & DAIR \\
\hline 532 & 83 & female & 32.9 & 3 & DAA & Gr.G Streptoccus & 2 & 107 & DAIR \\
\hline 572 & 75 & female & 26.2 & 3 & DAA & CNS, Propionibacterium acnes & 3 & 129 & 1-stage exchange \\
\hline 1236 & 73 & female & 27.3 & 2 & DAA & S. lugdunensis & 2 & 160 & DAIR \\
\hline 440 & 72 & female & 36.3 & 2 & DAA & $\begin{array}{l}\text { CNS, S. epidermidis, Enterococcus } \\
\text { faecalis, Propionibacterium sp. }\end{array}$ & 3 & 347 & 2-stage exchange \\
\hline 1162 & 47 & female & 19.1 & 2 & DAA & S. aureus & 3 & 386 & 1-stage exchange \\
\hline 835 & 83 & male & 29.6 & 2 & DAA & Finegoldia magna & 3 & 416 & 2-stage exchange \\
\hline 717 & 74 & male & 27.1 & 1 & DAA & Streptococcus sanguinis & 3 & 1431 & 1-stage exchange \\
\hline 883 & 63 & female & 45.6 & 2 & LAT & $\begin{array}{l}\text { S. epidermidis, Enterococcus } \\
\text { faecalis, S. warneri }\end{array}$ & 1 & 17 & DAIR \\
\hline 599 & 84 & male & 33.3 & 3 & LAT & S. epidermidis, Bacillus sp. & 1 & 17 & DAIR \\
\hline 109 & 73 & female & 29.0 & 2 & LAT & CNS & 1 & 17 & DAIR \\
\hline 1121 & 64 & male & 36.6 & 2 & LAT & Actinomyces sp. & 3 & 46 & DAIR \\
\hline 1100 & 65 & male & 40.3 & 3 & LAT & Streptococcus sp. & 2 & 238 & DAIR \\
\hline 453 & 81 & male & 28.3 & 3 & LAT & CNS & 3 & 306 & 1-stage exchange \\
\hline 84 & 74 & male & 27.7 & 1 & LAT & CNS & 3 & 1124 & 1-stage exchange \\
\hline
\end{tabular}

Abbreviations: ${ }^{\text {a }}$ : acute postoperative PJI, 2: acute hematogenous PJI, 3: chronic PJI, DAIR debridement and retention including exchange of mobile parts, PJI periprosthetic joint infection, $B M I$ body mass index, ASA American Society of Anesthesiologist score, UPI unique patient identification number, CNS coagulase negative staphylococci

In addition, in the DAA-group, the fraction of patients with polymicrobial infection was somewhat higher than in the LAT-group $(7 / 14$ vs $2 / 6, P=0.64)$. Six out of 7 polymicrobial PJI in the DAA-group were caused either by gramnegative bacilli or Enterococcus faecalis, which reflects the inguinal microbiome.

Patients with PJI had a higher BMI than those without (median 33.3, range 19 to 46 vs median 26.8, range 17 to 55 vs; $P<0.001)$ and a higher ASA class $(P<0.001)$. However, they did not differ in operation time (median 116 [70 to 223] vs median 109 [44 to 287]; $P=0.20$ ), gender $(P=0.83)$, age (median 74 [47 to 88 ] years vs median 71 [27 to 93] years; $P=0.24$ ) and blood loss (median 650 [200 to 1300] ml vs median 600 [30 to 3600 ] ml, $P=0.48$ ). Patients in the DAA group had a lower BMI $(P=0.01)$ as compared to the LAT-group (Table 1$)$.
In addition, they had less blood loss (median 500 [95 to 3600 ] ml vs median 700 [30 to 2800] $\mathrm{ml}, P<0.001$ ) and a shorter operating time (median 106 [44 to 236] minutes vs median 114 [ 49 to 287 ] minutes, $P<0.001$ ).

\section{Discussion}

The presence of an implant increases the risk for infection more than 100 '000fold [23, 24]. Therefore, the perioperative bacterial load around the incision site is a crucial risk factor for infection. Three years after switching from LAT to DAA as the routine approach, we observed a clustering of early infections with an unusual spectrum of microorganisms. We therefore asked the questions, whether in patients undergoing THA, the DAA, being located closer to the groin, would increase the rate of PJI, and whether the spectrum of 
microorganisms would differ in patients with the DAA as compared to those with the LAT-approach.

The published incidence of PJI after THA is 0.5 to $3 \%$ [1]. In patients with DAA, an incidence of up to $3 \%$ is described [13, 25]. Because patients undergoing debridement without exchange of mobile parts, as well as those with exclusive suppressive therapy might not appear in registers, the published infection rates may be underestimated [1]. We compared the rate of PJI in infection rate in patients with DAA vs those with LAT in our cohort of patients, including all consecutive patients undergoing elective THA and any kind of infection treatment. None of the suspected early exogeneous PJI was treated as a superficial infection with antibiotics alone. In case of wound healing disturbances, PJI was actively searched with an additional debridement and microbiological sampling. Our observed infection rate seems rather high but still it was within the published range. We controlled our clinical routines and found no mitigating factors that might contribute to a higher risk of infection [7]. Furthermore the rate of implant exchange due to PJI was comparable to register data thus the used DAA generally might not be considered as risk factor for PJI [26, 27]. But larger RCT trials should be performed to compare the DAA to established lateral and posterior approaches.

In our series, $87.0 \%$ of the patients with PJI had either acute or chronic exogenous infection independent from the approach. Thus, intraoperative contamination or implant contamination in the early postoperative time was by far the most frequent mechanism of infection. The overall infection rate was not different in the two groups. Thus, in contrast to published data [26] the introduction of the new approach was rather safe, which is probably due to the fact that the first 100 cases with the new incision site were operated by the same two experienced surgeons [15]. Exogenous polymicrobial infections were somewhat more frequent in the DAA- as compared to the LAT-group $(50.0 \%$ vs $33.3 \%, P=0.64)$, however, this difference was statistically not significant. Since in our previous cohort study of PJI from 1984-2001, the fraction of patient with polymicrobial PJI was only $12.7 \%(8 / 63)$ [28] in patients treated with the LAT approach, the DAA might be a risk factor for polymicrobial PJI in a larger cohort. Polymicrobial PJI are mainly observed in patients with wound healing disturbances. Thus, these infections are acquired during the early postoperative period. The risk of polymicrobial PJI might be lowered for DAA by a more lateral skin incision keeping more distance to the groin and the use of modern dressing techniques avoiding skin folds that might cause sweating and contamination. In addition, this modified approach reduces the risk for damaging the cutaneal femoral lateral nerve too [29]. We adopted this technique during the observation period after observing a clustering of infections and felt that there was no persistence of that problem.
The mean BMI and ASA score of the studied patients did not differ from published populations, indicating that our cohort represents a normal population of THA patients. As in previously published studies, patients with a higher BMI, a higher ASA score and longer operating time had a significantly increased risk of PJI in our entire cohort. These patients are at risk irrespective of the incision site $[12,13]$.

The main limitation of our study is the lack of randomization concerning the approach. A possible selection bias was introduced by operating 34 patients with the lateral approach, during the period when we already switched to the DAA. Five of the patients treated with the LAT-approach during the DAA-period suffered from PII, three of them had skin irritations in the groin making DAA not suitable. This might be confounding, and it cannot be excluded that the infection rate of the DAA group might have been higher, if all consecutive patients were operated with the DAA. An additional limitation is the relatively low number of patients, as compared to register studies with several thousands of patients. Thus, the study is likely to be underpowered leading to a lack of significant differences. The increased risk for gramnegative and polymicrobial infection should be verified, when data from larger cohorts will be available. At the present time, such data are missing, since minimal invasive approaches have been popularized only recently in Europe and the US. Strengths of our study are the complete follow-up of all patients, the completeness of data on ASA, BMI and causative microorganisms and the absence of undiagnosed PJI due to unrecognized suppressive therapy without thorough diagnostic work-up. This is an advantage in comparison to register data, which may not include all infected patients, because patients with antibiotic suppression or debridement might not be reported [1]. Another strength is the availability of a specialized interdisciplinary team caring for all patients with orthopedic infection. Thus, the risk for underreporting the early and mid-term infection risk of our patients is very low.

\section{Conclusions}

The introduction of the DAA did not increase the risk of PJI. However a tendency for a higher fraction of polymicrobial and gramnegative PJI was observed in patients treated with the DAA.

\footnotetext{
Abbreviations

ASA: American Society of Anesthesiologist; ASIS: Anterior superior iliac spine; BMI: Body mass index; CNS: Coagulase negative staphylococci; DAA: Direct anterior approach; DAIR: Debridement and implant retention; IDSA: Infections Disease Society on America; LAT: Lateral transgluteal approach; PJI: Periprosthetic joint infection; RA: Rheumatoid Arthritis; THA: Total hip arthroplasty; UPI: Unique patient identification number
}

Acknowledgements No Acknowledgements. 


\section{Funding}

No funding.

\section{Availability of data and materials}

Data will not be shared. We are not allowed to upload the data table due to restrictions by local authorities and data protection.

\section{Authors' contributions}

TI: study conception, writing of first draft, manuscript revisions, final approval of manuscript; WZ: data analysis, writing and revision of manuscript, final approval of manuscript; LB: data analysis, statistical analysis, final approval of manuscript; PG: data analysis, writing and revision of manuscript, final approval of manuscript; MC: study conception, data analysis, writing and revision of manuscript, final approval of manuscript.

\section{Competing interests}

The authors declare that they have no competing interests.

\section{Consent for publication}

Not applicable.

\section{Ethics approval and consent to participate}

Ethical approval was obtained by the local ethics committee (Northwest and Central Switzerland, EKNZ 2015-426). In accordance with the Ethics Committee, no specific informed consent was required.

\section{Author details}

'Interdisciplinary Unit for Orthopedic Infections, Kantonsspital Baselland, Rheinstrasse 26, 4410 Liestal, Switzerland. ${ }^{2}$ Leonardo, Hirslanden Klinik Birshof, Reinacherstrasse 28, 4142 Münchenstein, Switzerland. ${ }^{3}$ Department for Orthopedics and Trauma Surgery, Kantonsspital Baselland, Rheinstrasse 26, 4410 Liestal, Switzerland.

Received: 14 June 2016 Accepted: 9 November 2016

Published online: 14 November 2016

\section{References}

1. Dale H, Skramm I, Lower HL, Eriksen HM, Espehaug B, Furnes O, Skjeldestad FE, Havelin LI, Engesaeter LB. Infection after primary hip arthroplasty: a comparison of 3 Norwegian health registers. Acta Orthop. 2011;82(6):646-54.

2. Parvizi J, Gehrke T, Chen AF. Proceedings of the international consensus on periprosthetic joint infection. Bone Joint J. 2013;95-B(11):1450-2

3. Dale H, Fenstad AM, Hallan G, Havelin LI, Furnes O, Overgaard S, Pedersen AB, Karrholm J, Garellick G, Pulkkinen P, Eskelinen A, Makela K, Engesaeter LB. Increasing risk of prosthetic joint infection after total hip arthroplasty. Acta Orthop. 2012;83(5):449-58

4. Kurtz SM, Lau E, Schmier J, Ong KL, Zhao K, Parvizi J. Infection burden for hip and knee arthroplasty in the United States. J Arthroplasty. 2008;23(7):984-91.

5. Gundtoft PH, Overgaard S, Schonheyder HC, Moller JK, KjaersgaardAndersen P, Pedersen AB. The "true" incidence of surgically treated deep prosthetic joint infection after 32,896 primary total hip arthroplasties: a prospective cohort study. Acta Orthop. 2015;86(3):326-34

6. Huotari K, Peltola M, Jamsen E. The incidence of late prosthetic joint infections: a registry-based study of 112,708 primary hip and knee replacements. Acta Orthop. 2015;86(3):321-5

7. Alijanipour P, Heller S, Parvizi J. Prevention of periprosthetic joint infection: what are the effective strategies? J Knee Surg. 2014;27(4):251-8.

8. Grice EA, Segre JA. The skin microbiome. Nat Rev Microbiol. 2011;9(4):244-53.

9. Lindgren V, Garellick G, Karrholm J, Wretenberg P. The type of surgical approach influences the risk of revision in total hip arthroplasty: a study from the Swedish Hip Arthroplasty Register of 90,662 total hipreplacements with 3 different cemented prostheses. Acta Orthop. 2012:83(6):559-65.

10. Hodgkiss-Harlow KD, Bandyk DF. Antibiotic therapy of aortic graft infection: treatment and prevention recommendations. Semin Vasc Surg. 2011;24(4):191-8.

11. Reina N, Delaunay C, Chiron P, Ramdane N, Hamadouche M, t. Societe francaise de chirurgie orthopedique et. Infection as a cause of primary total hip arthroplasty revision and its predictive factors. Orthop Traumatol Surg Res. 2013;99(5):555-61.
12. Carroll K, Dowsey M, Choong P, Peel T. Risk factors for superficial wound complications in hip and knee arthroplasty. Clin Microbiol Infect. 2014;20(2):130-5.

13. Russo MW, Macdonell JR, Paulus MC, Keller JM, Zawadsky MW. Increased Complications in Obese Patients Undergoing Direct Anterior Total Hip Arthroplasty. J Arthroplasty. 2015;30(8):1384-7.

14. Matta JM, Shahrdar C, Ferguson T. Single-incision anterior approach for total hip arthroplasty on an orthopaedic table. Clin Orthop Relat Res. 2005:441:115-24.

15. Ilchmann T, Gersbach S, Zwicky L, Clauss M. Standard Transgluteal versus Minimal Invasive Anterior Approach in hip Arthroplasty: A Prospective, Consecutive Cohort Study. Orthop Rev (Pavia). 2013;5(4):e31.

16. Smith-Petersen MN. THE CLASSIC: Evolution of Mould Arthroplasty of the Hip Joint. Clin Orthop Relat Res. 2006;453:17-21.

17. Bauer R, Kerschbaumer F, Poisel S, Oberthaler W. The transgluteal approach to the hip joint. Arch Orthop Trauma Surg. 1979;95(1-2):47-9.

18. Ricciardi BF, Bostrom MP, Lidgren L, Ranstam J, Merollini KM, W-Dahl A. Prevention of surgical site infection in total joint arthroplasty: an international tertiary care center survey. HSS J. 2014;10(1):45-51.

19. Osmon DR, Berbari EF, Berendt AR, Lew D, Zimmerli W, Steckelberg JM, Rao N, Hanssen A, Wilson WR, Infectious Diseases A. Society of, Executive summary: diagnosis and management of prosthetic joint infection: clinical practice guidelines by the Infectious Diseases Society of America. Clin Infect Dis. 2013:56(1):1-10.

20. Zimmerli W, Sendi P. Orthopaedic implant associated infections. In: Bennett JE, Dolin R, Blaser MJ, editors. Principles and Practice of Infectious Diseases. 8th ed. Philadelphia: Elsevier; 2015. p. 1328-40.

21. Zimmerli W, Trampuz A, Ochsner PE. Prosthetic-joint infections. N Engl J Med. 2004;351(16):1645-54.

22. Zimmerli W, Clauss M. Periprosthetic Joint Infections after Total Hip and Knee Arthroplasty. In: Zimmerli W, editor. Bone and Joint Infections. From Microbiology to Diagnostics and Treatment. Chichester: Wiley Blackwell; 2015. p. $131-50$

23. Zimmerli W, Waldvogel FA, Vaudaux P, Nydegger UE. Pathogenesis of foreign body infection: description and characteristics of an animal model. J Infect Dis. 1982;146(4):487-97.

24. Zimmerli W, Sendi P. Pathogenesis of implant-associated infection: the role of the host. Semin Immunopathol. 2011;33(3):295-306.

25. De Geest T, Vansintjan P, De Loore G. Direct anterior total hip arthroplasty: complications and early outcome in a series of 300 cases. Acta Orthop Belg. 2013;79(2):166-73.

26. Spaans AJ, van den Hout JA, Bolder SB. High complication rate in the early experience of minimally invasive total hip arthroplasty by the direct anterior approach. Acta Orthop. 2012;83(4):342-6.

27. Leunig M, Faas M, von Knoch F, Naal FD. Skin crease 'bikini' incision for anterior approach total hip arthroplasty: surgical technique and preliminary results. Clin Orthop Relat Res. 2013;471(7):2245-52.

28. Giulieri SG, Graber P, Ochsner PE, Zimmerli W. Management of infection associated with total hip arthroplasty according to a treatment algorithm. Infection. 2004:32(4):222-8.

29. Ropars M, Morandi X, Huten D, Thomazeau H, Berton E, Darnault P. Anatomical study of the lateral femoral cutaneous nerve with special reference to minimally invasive anterior approach for total hip replacement. Surg Radiol Anat. 2009;31(3):199-204.

\section{Submit your next manuscript to BioMed Central and we will help you at every step:}

- We accept pre-submission inquiries

- Our selector tool helps you to find the most relevant journal

- We provide round the clock customer support

- Convenient online submission

- Thorough peer review

- Inclusion in PubMed and all major indexing services

- Maximum visibility for your research

Submit your manuscript at www.biomedcentral.com/submit
Biomed Central 